\title{
Antiquité et Révolution française dans la pensée et les lettres allemandes à la fin du XVIII ${ }^{\mathrm{e}}$ siècle
}

\section{Lucien Calvié}

\section{(2) OpenEdition \\ 1 Journals}

\section{Édition électronique}

URL : https://journals.openedition.org/ahrf/268

DOI : $10.4000 /$ ahrf.268

ISSN : 1952-403X

Éditeur :

Armand Colin, Société des études robespierristes

\section{Édition imprimée}

Date de publication : 1 septembre 1999

Pagination : 455-475

ISSN : 0003-4436

\section{Référence électronique}

Lucien Calvié, «Antiquité et Révolution française dans la pensée et les lettres allemandes à la fin du xvIII siècle », Annales historiques de la Révolution française [En ligne], 317 | juillet-septembre 1999, mis en ligne le 10 avril 2006, consulté le 23 avril 2022. URL : http://journals.openedition.org/ahrf/268 ; DOI : https://doi.org/10.4000/ahrf.268

Ce document a été généré automatiquement le 23 avril 2022.

Tous droits réservés 


\title{
Antiquité et Révolution française dans la pensée et les lettres allemandes à la fin du XVIII ${ }^{\mathrm{e}}$ siècle
}

\author{
Lucien Calvié
}

1 La question du discours allemand, littéraire et philosophique, sur l'Antiquité comme discours, en deuxième instance, sur la Révolution française, est peu abordée, à notre connaissance, dans les travaux de recherche actuels. Elle a cependant un long et riche passé théorique, en particulier chez Georg Lukacs, marxiste hongrois de culture largement allemande, dans ses études des années 1930 sur la littérature classique allemande (Goethe, Schiller et Hölderlin), à l'époque de son deuxième et long séjour à Moscou à partir de 1933, par exemple dans son article de 1935, paru en 1937, sur « la théorie de la littérature moderne chez Schiller », à propos de Georg Forster comme du jeune Hegel ${ }^{1}$. Dans ce même article, Lukacs s'appuie explicitement sur l'idée d'» illusion héroïque » historiquement nécessaire qu'il emprunte au jeune Marx ; il cite d'ailleurs le passage célèbre de La Sainte Famille de 1845 :

«Robespierre, Saint-Just et leur parti ont succombé parce qu'ils ont confondu

l'antique république, réaliste et démocratique, qui reposait sur les fondements de

l'esclavage réel, avec l'État représentatif moderne, spiritualiste et démocratique,

qui repose sur l'esclavage émancipé, la société ${ }^{2}$. "

Un disciple roumain, puis français, de Lukacs, Lucien Goldmann, bien que plus intéressé par les textes de jeunesse du maître (La Théorie du roman de 1914-1920 et Histoire et Conscience de classe de 1923) que par ses écrits de Moscou (3), reprend la discussion amorcée par Marx, puis par Lukacs, en la condensant et en la systématisant, dans un passage de Sciences humaines et Philosophie en 1952, au sujet du lien problématique entre la société bourgeoise moderne en cours de constitution et la puissance de la référence antique dans les lettres et la pensée :

«[...] l'humanisme de la Renaissance, l'énorme importance qu'a prise la culture grecque pour la pensée d'Europe occidentale s'expliquent par le fait qu'une société bourgeoise orientée, non plus vers l'au-delà, mais vers l'homme et vers la terre, s'était dégagée au sein de l'ancienne société féodale et qu'elle trouvait dans les 
écrits et les œuvres de l'Antiquité gréco-latine une culture et un art orientés, eux aussi, vers la terre et, surtout, vers l'homme. Ce sont leurs propres aspirations et leur propre mentalité que les humanistes retrouvaient dans les écrits de Platon et de Cicéron et ils parlaient grec et latin en attendant que le développement de la société bourgeoise leur permît de dire la même chose dans leur langue nationale. Si, d'ailleurs, l'Antiquité a continué si longtemps à avoir, pour l'Allemagne, une importance existentielle (il suffit de penser à Goethe, Hegel, Hölderlin, Nietzsche), c'est précisément parce que la faiblesse de la bourgeoisie, le faible développement $\mathrm{du}$ capitalisme, l'absence d'une révolution bourgeoise, n'ont pas permis aux penseurs allemands d'abandonner l'Antiquité pour parler leur propre langue comme l'ont fait les idéologues du tiers état en France ou en Angleterre. À la fin du XIXe siècle, l'Antiquité devint, en Allemagne comme partout, et plus encore, un ensemble de connaissances scolaires et universitaires ${ }^{4}$. »

C'est au fond le thème récurrent du retard économico-politique allemand, ou de la «misère allemande» que reprend ici Goldmann: la bourgeoisie allemande "parle antique", par la bouche d'écrivains comme Goethe, Schiller ou Hölderlin, aussi longtemps qu'elle est trop faible pour "parler allemand », c'est-à-dire pour constituer son État national.

4 Un germaniste français enfin, le regretté Pierre Bertaux, aborde également la question dans son livre de 1969 - la date n'est pas sans importance - sur Hölderlin et la Révolution française; Bertaux remarque que la critique universitaire de langue allemande - à l'exception de Lukacs précisément, comme il le souligne ${ }^{5}$ - n'a pas été capable d'apercevoir le lien, chez le poète, entre ses opinions «jacobines »- ou plus exactement "girondines", selon Bertaux - et son hellénisme, si caractéristique des grands poèmes comme du roman Hypérion de 1797-1799. De plus, selon Bertaux, le " pathos» antiquisant de Hölderlin est dans la dépendance, y compris stylistique, de celui, tout aussi antiquisant, des révolutionnaires français, de ces journaux et pamphlets français dont Hölderlin, tout comme Hegel, a pu lire de nombreux exemplaires en Allemagne même ${ }^{6}$.

5 La question que nous voudrions aborder ici - et la remarque de Bertaux citée ci-dessus le montre - se situe au point d'intersection de trois questions classiquement répertoriées et envisagées d'ordinaire par des chercheurs relevant de spécialités différentes - germanistes, historiens de l'art, historiens, voire philosophes : la question, tout d'abord, du rapport de la Révolution française à l'Antiquité dans ses formes extérieures, y compris artistiques, mais aussi dans le discours et l'idéologie ${ }^{7}$, surtout autour de l'idée républicaine, de ses composantes (la vertu, le bien et le salut publics, la patrie) et de ses antithèses (le despotisme, la tyrannie) ; la question, ensuite, du rapport des lettres et de la pensée allemandes à l'Antiquité et à ses idéaux, principalement autour d'un "classicisme allemand» chronologiquement identifié à la période de l'amitié productive entre Goethe et Schiller, de 1794 à 1805, classicisme qu'une puissante tradition critique présente encore souvent comme le produit d'une rupture, non seulement avec une Révolution française " excessive » et " terroriste ", mais aussi avec toute réalité politique immédiate, impure et laide par nature. Rares encore sont aujourd'hui ceux qui osent écrire, par exemple, que « tous les textes de Schiller après 1789 » sont « une réaction aux événements de la Révolution française ${ }^{8}$ "; la question enfin, désormais largement étudiée, en R.D.A. dès les années 1950 et 1960 puis en R.F.A. à partir de 1968-1970, et enfin, récemment, à l'occasion du bicentenaire de 1789, des réactions politiques et intellectuelles à la Révolution française à l'intérieur du SaintEmpire et des États allemands 9. 
6 L'étude systématique du discours allemand sur l'Antiquité comme discours sur la Révolution française suppose la mise en œuvre de moyens matériels et intellectuels importants, dans un projet collectif réunissant des historiens, des historiens de l'art, des philosophes et des germanistes. Nous nous contenterons de poser ici, comme esquisse de ce projet, quelques jalons significatifs, sans aucune prétention à l'exhaustivité. C'est ainsi, par exemple, que les importants écrits de Wilhelm von Humboldt sur la Révolution française, l'État et la Grèce antique, échelonnés de 1791-1792 à 1808-1810, sont absents de notre étude. Nous nous proposons de revenir ultérieurement de manière détaillée sur ce théoricien.

Winckelmann et le néo-classicisme

7 Le discours allemand sur l'Antiquité comme discours sur la Révolution française s'inscrit dans un phénomène plus ancien, remontant au milieu du XvIII siècle, celui du néo-classicisme artistique. Et le classicisme littéraire allemand, des années 1790 à la mort de Schiller en 1805 - précédé dans les histoires de la littérature par un "préclassicisme » (Winckelmann, Lessing) et par le "Sturm und Drang » des années 1770 (Herder, le jeune Goethe)-, n'est jamais, au fond, chronologiquement et thématiquement, qu'un néo-classicisme en littérature

8 Pour Jean Starobinski, si "l'année 1789 est une ligne de partage dans l'histoire politique de l'Europe [...], on ne peut y situer aucun événement majeur de l'histoire de l'art [...] Le "retour à l'antique » précède la Révolution, le goût néo-classique s'est affirmé, puis largement diffusé à partir de $1750^{10}$. "

Dans un livre sur le néo-classicisme paru en 1968, l'historien de l'art Hugh Honour examine les liens entre le néo-classicisme et les revendications politiques éclairées et libérales, avant 1789, en particulier à propos du peintre David et de ses tableaux Le Serment des Horaces de 1784 et Brutus, commencé en $1788{ }^{11}$. Même si les jacobins, comme le note Honour, «firent du néo-classicisme leur style artistique officiel tandis que le culte de l'Antiquité devenait presque une religion ${ }^{12}$ ", la question peut se poser à propos du rapport néo-classicisme-Révolution comme à propos du rapport LumièresRévolution : la succession chronologique implique-t-elle un lien de cause à effet? La question des origines, en particulier intellectuelles, de la Révolution française est complexe et elle a été envisagée de bien des manières. S'il faut refuser la vision d'un engendrement en quelque sorte mécanique de la Révolution par ce qui la précède dans l'ordre intellectuel (les Lumières) et artistique (le néo-classicisme), il reste que ces trois éléments - les Lumières, le néo-classicisme et la Révolution - constituent ce que l'on pourrait appeler un ensemble significatif et dynamique dont le sens est donné, téléologiquement, par 1789. Lukacs, d'ailleurs, ne disait pas autre chose lorsqu'il écrivait dans l'article de 1935-1937 déjà cité :

«Le modèle antique a été l'idéal politique nécessaire de la classe bourgeoise en lutte pour son autonomie et le pouvoir politique. La Polis s'est érigée de plus en plus nettement en modèle politique des révolutionnaires bourgeois jusqu'à ce que cette évolution trouve dans la Grande Révolution française son accomplissement pratique ${ }^{13}$."

10 Bien des textes néo-classiques (du point de vue de la théorie de l'art) et pré-classiques ou classiques (du point de vue traditionnel de l'histoire littéraire) publiés en Allemagne avant 1789 peuvent se lire dans la perspective qui vient d'être esquissée. C'est le cas de ceux de Winckelmann, aussi bien les Pensées sur l'Imitation des cuvres grecques de 1755 que l'Histoire de l'art de l'Antiquité une dizaine d'années plus tard. En posant, dans le 
texte de 1755 , le principe, non d'une imitation mécanique des statues grecques, mais d'une appropriation de la pensée, ou de l'idéal, et de l'existence physique, ou de la nature, des Grecs anciens, Winckelmann liait le sentiment du beau à une situation humaine générale: le sol, la végétation, le climat, mais aussi le cadre social et historique dans lequel les Grecs avaient créé leurs œuvres. Or, cette société et cette histoire sont, pour Winckelmann, faites de liberté. Le mot ne figure pas vraiment, du moins comme notion explicitement politique, dans les Pensées sur l'Imitation, mais l'auteur y souligne un contraste, promis à une longue fortune chez les théoriciens de l'art jusqu'à Hegel inclus, entre un art égyptien enserré dans une législation stricte et une Grèce où la "belle nature " peut se développer dans le plaisir grâce à la « liberté des mœurs »; et il est question quelques lignes plus loin de la «belle humanité » des Grecs dans sa «florissante liberté » ${ }^{14}$.

11 L'argumentation de Winckelmann prend un tour plus nettement politique dans l'Histoire de l'art de l'Antiquité de 1764, autour de la notion de liberté :

« Du point de vue de la constitution et du gouvernement de la Grèce, la liberté est la cause la plus éminente de l'avantage dont l'art y jouit. De tout temps la liberté a eu son siège en Grèce, même auprès du trône des rois qui gouvernaient de façon paternelle, avant même que les lumières de la raison ne permettent aux Grecs de goûter la douceur d'une liberté pleine et entière [...] C'est grâce à la liberté que s'est élevée, comme un noble rameau sur un tronc sain, la pensée du peuple tout entier ${ }^{15}$. »

Wilhelm Heinse et la voie italienne

Cette association entre la beauté, naturelle et artistique, le bonheur, physique et moral, et la liberté, celle des citoyens et celle de la cité qu'ils constituent, caractérise selon Winckelmann la Grèce ancienne. Il souhaite, à travers l'éducation artistique par l'imitation bien comprise, en retrouver le secret et en renouveler le miracle dans le monde moderne. Cette aspiration à la fois artistique et politique traverse tout le deuxième $\mathrm{XVIII}^{\mathrm{e}}$ siècle allemand, jusqu'à la période révolutionnaire elle-même, sous des formes diverses. On en trouve par exemple l'écho, en 1785-1787, dans le roman utopique de Wilhelm Heinse Ardinghello et les îles bienheureuses. Un peintre italien du $\mathrm{XVI}^{\mathrm{e}}$ siècle fonde avec des compagnons un État idéal dans des îles de la mer Égée. La liberté la plus complète, physique, morale, artistique et politique y est le règle et le fondement du bonheur individuel et collectif.

Le cadre géographique et artistique largement italien de ce roman est l'occasion de signaler un phénomène important: c'est presque exclusivement à travers des références italiennes - l'archéologie à Herculanum et Pompéi ou à Rome, les musées, surtout celui du Vatican, mais aussi la beauté du ciel et des paysages, l'atmosphère de liberté et de bonheur qui, pour les voyageurs allemands, paraît émaner du peuple - que s'effectue le contact des penseurs et hommes de lettres allemands avec l'Antiquité grecque. Winckelmann, Heinse, Goethe à deux reprises - y compris en Sicile, terre marquée d'hellénisme - et Herder font des voyages et même de longs séjours en Italie des années 1760 (Winckelmann) aux années 1780 (les trois autres); aucun ne connaît directement la Grèce, son sol et ses habitants. Mais cette Italie qu'ils découvrent, avec ses vestiges antiques, avec sa nature et son peuple qu'ils trouvent si beaux, jusque dans la misère, représente pour eux, globalement, la présence visible et sensible de l'Antiquité, y compris grecque, dans le monde moderne. Goethe a avoué, sur le tard, que la seule période vraiment heureuse de son existence fut celle des séjours italiens, à partir de 1786. Ce n'était pas seulement dire l'étroitesse étouffante de la cour et de 
l'administration de Weimar, c'était peut-être aussi exprimer un besoin de beauté, de bonheur et de liberté qui était aussi un besoin politique partagé avec nombre de ses contemporains et compatriotes, de Winckelmann à Herder et à Hölderlin.

$\mathrm{Au}$ début de la troisième partie du roman, Heinse insère un long développement sur la société et l'État. Revenant sur la distinction, classique depuis l'Antiquité, entre démocratie, aristocratie et monarchie, il considère comme inévitable leur dégénérescence, respectivement en anarchie, oligarchie et despotisme, les formes intermédiaires et vertueuses imaginées par les théoriciens étant intenables dans la pratique. Le personnage qui s'exprime, Frescobaldi, déclare préférer à ces constructions abstraites la réalité historique des cités antiques :

«[...] Je préfère pour ma part m'en tenir à Rome et à Sparte, vieux États plus nobles et plus parfaits que tous ceux que nous connaissons, peut-être que tous ceux qui ont jamais existé. Quiconque est engagé dans les luttes politiques [...] doit lire leur histoire, en pénétrer le sens profondément en le confrontant avec son expérience, et il en tirera une tout autre lumière qu'il ne le ferait des plus sages prescriptions d'un écrivain politique isolé ${ }^{16}$.»

15 Ces considérations sont précédées d'une critique radicale du despotisme, forme dégénérée de la monarchie, le despote étant décrit comme « une vermine, un ténia logé dans le corps ${ }^{17}$ ", et de l'oligarchie, forme dégénérée de l'aristocratie, comparée à une " hydre du despotisme à plusieurs têtes, une vermine sur le corps, non plus unique mais multiple ${ }^{18} "$, avec cette conclusion fort claire :

«Un État d'hommes dignes de ce nom, un État entièrement fait pour tous et pour chacun, ne peut au fond être qu'une démocratie ${ }^{19}$. "

16 Et Heinse de donner, par Frescobaldi interposé, comme exemple de l'heureuse évolution d'un peuple vers le «bien de l'ensemble », le bien public, le peuple romain qui, en matière politique, « dans cet art primordial, l'emporte sur toutes les nations » et a su, par un long effort historique, «se débarrasser de la puissance de ses rois et ensuite, avec ses tribuns [...], dompter l'aristocratie ${ }^{20}$.»

17 N'est-ce pas déjà un révolutionnaire français admirateur de la Rome républicaine que l'on croirait lire, un Saint-Just avant la lettre : modèles spartiate et romain connus et médités - «le monde est vide depuis les Romains et leur mémoire le remplit, et prophétise encore la liberté", écrivait Saint-Just ${ }^{21}$ - et supériorité absolue du républicanisme démocratique, garantie du bien public et du bonheur individuel, sur les formes nécessairement dégénérées de la monarchie et de l'aristocratie : le despotisme et l'oligarchie haïs?

Schiller, avant et après 1789

18 Au printemps 1788 Schiller publie dans la revue Le Mercure allemand de Wieland un poème intitulé Les Dieux de la Grèce qui fait sensation et même scandale par son paganisme hellénisant. En vingt-cinq strophes, il oppose au monde moderne et chrétien, décrit comme malheureux, prosaïque, divisé, soumis et laid, le monde ancien et disparu de la Grèce antique, présenté, en contraste, comme heureux, poétique, harmonieux, libre et beau. Les dieux grecs, unis à la fois à la nature et à l'humanité par une foule de divinités intermédiaires et de héros, se trouvent opposés au Dieu du monothéisme, radicalement séparé des hommes et leur imposant ses lois aussi inexorablement que la science moderne impose les siennes, par exemple celle de la gravitation, à une nature désormais inanimée car privée de ses dieux. Les Grecs étaient 
plus divins que les contemporains de Schiller car leurs dieux étaient plus humains que le Dieu chrétien.

Cette longue élégie nostalgique n'exprime aucune vue explicitement politique mais il faut la comprendre dans le cadre général esquissé plus haut de l'hellénisme allemand de la deuxième moitié du xvIII ${ }^{e}$ siècle, pour lequel la liberté (individuelle et politique), le bonheur (individuel et collectif) et la beauté (naturelle et artistique) ne font qu'un. Et c'est bien ainsi que le poème est compris en son temps.

D'une part, le comte Friedrich Leopold von Stolberg attaque Schiller en août 1788 dans la revue Musée allemand de Leipzig: son poème, selon lui, n'est qu'un long blasphème immoral et antichrétien; il fait l'éloge d'une prétendue religion grecque qui n'est, en fait, qu'» idolâtrie la plus grossière et athéisme le plus triste ${ }^{22}$ ». D'autre part, en mai 1789, dans la revue Nouvelles Études sur la littérature et les peuples paraissant également à Leipzig, un article anonyme prend la défense du poème en distinguant la question de la création poétique reconstruisant par l'imagination un monde disparu, celui de la Grèce antique et de ses dieux si humains, et la question de la croyance religieuse positive, personne ne pouvant croire que Schiller, comme feint de le croire Stolberg, a foi en les dieux antiques. Au fond, selon le recenseur, le poème de Schiller a le mérite d'offrir à son lecteur les moyens de comprendre le monde contemporain. On sait que l'auteur de cette recension parue sans nom d'auteur était Georg Forster, le futur «jacobin » mayençais de $1792{ }^{23}$.

Dès le texte de 1788 des Dieux de la Grèce, cependant, et beaucoup plus clairement dans la deuxième version du poème, moins nettement antichrétienne et publiée en 1803 mais sa rédaction est bien antérieure-, se fait jour chez Schiller une tendance à l'intériorisation intellectuelle, à l'opposition insurmontable entre l'inaccessible idéal et l'inacceptable réalité, tendance qui ira s'accentuant, en tendant au pessimisme, au cours des années 1790 et jusqu'aux premières années du XIX siècle :

«Bel univers, où es-tu? Reviens / Âge charmant et fleuri de la nature. Ah, c'est seulement dans le pays feérique des chants / Que vit encore ta trace dorée ${ }^{24}$. "

Seul l'art, donc, est en mesure de préserver les signes du bel univers antique, désormais irrémédiablement disparu.

D'une autre manière, philosophiquement plus élaborée et marquée par le contact studieux avec la philosophie de Kant, les Lettres sur l'Éducation esthétique publiées par Schiller en 1795 témoignent de la même tendance idéaliste et pessimiste. Face à l'événement révolutionnaire français, Schiller est réticent dès 1789-1790 et la radicalisation française des années 1792-1794 vient seulement en renfort de cette réticence initiale. Dans les Lettres de 1795, il cherche à déterminer les conditions d'un changement progressif, esthétique, moral, intellectuel et enfin politique, qui rendrait inutile la brutalité du processus révolutionnaire tel qu'il s'est développé en France et il insiste sur la nécessité, pour l'humanité moderne, de retrouver " par un art supérieur ${ }^{25}$ »- qui peut bien, finalement, avoir le sens d'une révolution-, ce que les Grecs de l'Antiquité, selon lui, les Athéniens surtout, réalisaient de façon naturelle et spontanée, " naïve » si l'on préfère, pour reprendre le vocabulaire schillérien : l'harmonie entre la liberté de chacun et celle de tous, l'équilibre heureux et beau de la cité et de l'individu, ou du citoyen. Mais cette liberté ainsi définie n'est pas très différente de cette "liberté des Anciens ", collective, politique et héroïque que Benjamin Constant opposera, en 1814 et 1819, dans De l'Esprit de conquête et de l'usurpation puis dans De la Liberté des Anciens comparée à celle des Modernes, à la « liberté des Modernes ", individuelle, civile et 
jouisseuse. Schiller semble croire encore possible la renaissance, dans le monde moderne, de cette liberté antique, par une autre voie que celle, illusoire et violente selon lui, de la Révolution française, alors que Constant l'estimera impossible et même dangereuse, allant même jusqu'à accuser la volonté de la faire revivre d'avoir en partie fondé la Terreur robespierriste. En un sens, et paradoxalement, le Schiller des Lettres de 1795, par son désir de faire revivre la belle liberté des Anciens, n'est pas, dans la perspective définie par Constant en 1814 et 1819, très éloigné d'un Robespierre et d'un Saint-Just. Même dans son refus de la voie révolutionnaire française, Schiller n'est pas du tout étranger à l'un des éléments essentiels de l'idéologie de la Révolution : le retour à l'admirable liberté supposée des anciennes républiques et des anciennes cités « illusion héroïque », selon le jeune Marx de 1845.

Forster et Herder

En 1788, on l'a vu, Forster avait pris la défense du poème de Schiller sur les dieux grecs contre la réaction politico-religieuse représentée par Stolberg. Mais, alors que Schiller, au cours des années 1790 , évolue vers des positions intellectuelles et politiques modérées et développe un idéalisme subjectif et esthétisant qui constitue un élément de ce qu'on appelle le "classicisme allemand ", la réflexion de Forster, au contraire, se radicalise. Parallèlement, sa vision de l'Antiquité s'écarte de celle de Schiller et devient plus directement politique. Dès 1789 , dans un essai intitulé L'Art et l'époque et publié dans la revue Thalia dirigée par Schiller, Forster, reprenant certaines des thèses de Winckelmann, mais aussi des Dieux de la Grèce de Schiller, oppose une image harmonieuse et belle de la Grèce antique à une actualité politique allemande décevante. Dans son article de 1935 sur Schiller, Lukacs cite longuement, pour montrer le caractère plus politique et radical de la " grécomanie » du futur « jacobin » mayençais de 1792, en comparaison de celle de Schiller, un extrait des Vues du Rhin inférieur, récit de voyage publié en 1791-1792 dans lequel Forster oppose la beauté majestueuse des œuvres de l'art grec (les statues de Phidias par exemple), présentée comme correspondant à la grandeur morale et politique des anciens Grecs, à la médiocrité des artistes et littérateurs de son temps, signe, selon lui, de la faiblesse morale et politique, de la « misère » moderne des Allemand ${ }^{26}$.

La position de Forster n'est pas sans parenté, parfois, avec celle de Herder. Celui-ci, souvent présenté, et certes avec raison, comme un de ceux qui, avec le jeune Goethe, dans le Sturm und Drang du début des années 1770, ont fait naître l'intérêt pour le Moyen Âge et un art "gothique » supposé spécifiquement germanique, est cependant fortement marqué par l'influence de Winckelmann dont il a d'ailleurs publié deux éloges, l'un en 1777 et l'autre en 1781, dans Le Mercure allemand de Wieland ${ }^{27}$. Il s'intéresse à l'Antiquité de bien des façons, avec le désordre, les contradictions, mais aussi la richesse de vues qui le caractérisent. Dès 1774, dans Une autre Philosophie de l'histoire, reprenant l'opposition soulignée par Winckelmann entre une Égypte asservie et une Grèce libre, mais en la discutant et en la relativisant, il fait l'éloge de la Grèce antique, beauté et liberté unies, et voit dans la cité grecque l'union harmonieuse de la liberté individuelle et de la discipline patriotique ${ }^{28}$. Et il ajoute :

«La Grèce devint le berceau du sentiment d'humanité, de l'amour des peuples, de la belle législation, de ce qu'il y a de plus agréable dans la religion, les mœurs, le style, la poésie, les coutumes et les arts. Tout était joie juvénile, grâce, jeu et amour ${ }^{29}$ ! » développe un point de vue qui, en apparence, contredit celui de Winckelmann : aucune civilisation, aucun peuple n'est privilégié dans le tableau de l'évolution de l'humanité, 
aucun n'est un modèle politique ou artistique pour les autres peuples. Le livre XIII cependant, et contradictoirement, présente une fois encore l'Antiquité grecque avec enthousiasme, à la manière de Winckelmann, comme une période supérieurement heureuse et belle du point de vue de l'art, de la pensée et de la politique théorique et pratique :

Les hommes, comme réveillés de leur état de minorité (Unmündigkeit), apprirent à réfléchir par eux-mêmes à leur constitution politique. Et c'est ainsi que l'époque des républiques grecques fut le premier pas de l'esprit humain vers sa majorité (Mündigkeit) sur l'importante question de savoir comment des hommes peuvent être gouvernés par des hommes ${ }^{30}$.

Et Herder d'expliquer la genèse de la cité et de l'esprit grecs :

"Voici quel était le ton dominant de la poésie, de l'histoire et de la politique grecques: chaque citoyen devait connaître ses concitoyens et s'occuper périodiquement des affaires publiques auxquelles il ne pouvait se soustraire [...] Gouverner les hommes ou agir comme membre vivant de la société, c'était là le trait dominant de cette âme grecque en train de croître. Il ne faut donc pas s'étonner que la philosophie du penseur abstrait, elle aussi, ait eu pour but la formation des mœurs ou de l'État, comme le montrent les exemples de Pythagore, de Platon et même d'Aristote ${ }^{31}$.»

28 Mais Herder, sans doute conscient de la contradiction entre son principe général d'analyse historique - celui de l'égale dignité des civilisations apparues sur terre - et son éloge appuyé des cités grecques et de leur "âme», clôt son livre XIII sur des «remarques générales » qui démontrent que la brillante Antiquité grecque n'a jamais été que le résultat d'un déterminisme géographique et historique dont les circonstances ne sauraient se renouveler :

« Pourquoi les Grecs éclairés furent-ils au monde ? Parce qu'ils se trouvaient dans un tel lieu et une telle situation et que, dans de telles circonstances, ils ne pouvaient être rien d'autre que des Grecs éclairés ${ }^{32}$."

Caractère unique et non renouvelable, donc, du «miracle » grec, à la fois artistique, intellectuel et politique, mais aussi limites de la "grécomanie» de l'Aufklärung indiquées par l'un de ses meilleurs représentants et son meilleur critique : Herder.

La génération de 1770 : Friedrich Schlegel, antiquisant et « jacobin »

On retrouve chez le jeune Friedrich Schlegel des éléments de l'hellénisme radical et «jacobin » qui caractérisait Forster. Schlegel publie d'ailleurs en 1797 dans la revue Lycée des Beaux-Arts un éloge de Forster, mort à Paris en 1794. Il a l'audace de donner comme sous-titre à son texte « fragment d'une caractéristique des classiques allemands 33 ». Cela sonne comme une provocation à l'égard de Goethe et de Schiller qui, dès 1791-1792, ont marqué leurs distances à l'égard du « jacobin » mayençais ${ }^{34}$. Forster, lucidement, avait d'ailleurs analysé les motifs de la forte réticence de ses anciens amis :

«Ils [Goethe et Schiller] ne peuvent pas comprendre un homme qui est capable aussi d'agir en son temps et ils me prennent en horreur lorsque je mets en œuvre les principes qu'ils ont eux-mêmes, sur le papier, honoré de leur approbation ${ }^{35}$. » d'ailleurs - la " grécomanie » de celui-ci pour une forme de sa "gallomanie », c'est-àdire de son « jacobinisme»:

«À peine la fièvre froide de la gallomanie nous a-t-elle quittés / Qu'éclate la fièvre brûlante de la grécomanie. / La grécité, qu'était-ce ? Raison, mesure et clarté. C'est pourquoi, / Pensais-je : / un peu de patience, messieurs, avant de nous parler de grécité ${ }^{36}$.» 
L'hellénisme à tendance révolutionnaire et la "grécomanie » gallomane ou « jacobine » de Schlegel ${ }^{37}$ critiqués par Schiller s'expriment principalement dans son texte écrit en 1795 et publié en 1797 sous le titre Sur l'étude de la Poésie grecque ${ }^{38}$. Ce livre a d'abord été conçu comme le premier tome d'un grand ouvrage sur Les Grecs et les Romains qui ne vit jamais le jour. Dans ce texte, Schlegel considère que l'harmonie de la Grèce ancienne reposait sur l'unité de tous les domaines de l'activité humaine: politique, arts, philosophie et littérature. En Grèce, la beauté était l'affaire, non des spécialistes, mais du peuple tout entier, pour qui elle était inséparable de la politique, et donc de la liberté de tous et de chacun. En cela, par son sens politique, cette harmonie grecque reste un modèle pour le monde moderne. Schiller lui aussi, dans les Lettres sur l'Éducation esthétique de 1795, insiste sur l'unité harmonieuse des différentes sphères de l'activité humaine dans la Grèce antique. La perte de cette unité constitue même pour lui, dans la ligne du poème Les Dieux de la Grèce de 1788, la caractéristique principale d'un monde moderne divisé, à la fois par le monothéisme chrétien, le rationalisme scientifique et l'atomisation des fonctions sociales et économiques. Mais il considère cette belle harmonie grecque comme irrémédiablement révolue, le modèle qu'elle peut encore constituer ne valant plus que comme anticipation utopique du résultat d'un long processus éducatif, à la fois esthétique, moral et politique, et non, comme chez Schlegel, comme résultat immédiatement possible d'un changement révolutionnaire. La rupture entre les deux hommes n'avait donc pas pour seule cause une bien réelle rivalité littéraire, mais aussi une divergence intellectuelle et politique sur la valeur et le sens de la Révolution française, d'une part, et sur l'Antiquité grecque, d'autre part, c'est-à-dire sur la corrélation entre "gallomanie», ou «jacobinisme», et « grécomanie ».

En 1796, dans un long compte rendu de l'essai de Kant La Paix perpétuelle de 1795, Schlegel, favorable au radicalisme révolutionnaire français, développe, contre le républicanisme modéré du philosophe de Königsberg, qui n'est le plus souvent qu'une mise en forme théorique et nostalgique de l'absolutisme éclairé de Frédéric II de Prusse dans ses virtualités les plus libérales, l'idée d'un républicanisme nécessairement démocratique, plus radical, donc, que celui de la Constitution directoriale de l'an III dont se satisfaisaient aussi bien Kant que le « jacobin » cisrhénan Joseph Görres ${ }^{39}$.

Schlegel, né en 1772, appartient à une génération d'intellectuels allemands nettement plus jeunes que ceux dont nous avons jusqu'à présent mentionné les écrits. Winckelmann, Herder, Goethe, Heinse, Forster et Schiller ont vu le jour respectivement en 1717, 1744, 1749 (Goethe et Heinse), 1754 et 1759. Pour ces hommes, formés principalement par les différents courants de l'Aufklärung dans le cadre des États particuliers du Saint-Empire, l'événement révolutionnaire français représente, avec ses conséquences en pays de langue allemande, une crise générale qui ne les concerne qu’à un âge déjà assez avancé, à l'exception bien sûr de Winckelmann, mort en 1768. Les crises des années 1790 ébranlent le système politique et intellectuel qui les a formés, essentiellement l'Aufklärung et l'absolutisme plus ou moins éclairé des États particuliers. Cela vaut aussi pour Kant, né en Prusse Orientale en 1724, à Königsberg, où il meurt en 1804.

Pour la génération de Schlegel, par contre, qui n'a qu'une vingtaine d'années en 1789 ou au début des années 1790, et qui est celle du premier romantisme allemand (Novalis est né lui aussi en 1772, August Wilhelm Schlegel, le frère de Friedrich, en 1767, Tieck et Wackenroder en 1773 - ils sont donc les très exacts contemporains de "jacobins" 
allemands comme les kantiens Erhard et Bergk, nés en 1766 et 1769, Rebmann, né en 1768, et Görres, le plus jeune, né en 1776, mais aussi de Saint-Just, Hoche et Napoléon Bonaparte, nés respectivement en 1767, 1768 et 1769), la première grande expérience personnelle et politique est celle de la Révolution française et de ses répercussions multiples en pays allemand. La crise, le moment du choix décisif, pour ces jeunes gens, ce n'est pas le phénomène révolutionnaire dans sa globalité - cela, c'est leur univers en quelque sorte normal -, c'est la crise à l'intérieur de la Révolution elle-même ; non pas tant, du reste, la phase radicale et «terroriste » de 1792-1794, pour laquelle ils n'ont que sympathie et même enthousiasme - la patrie en danger, le tyrannicide sous la forme du procès et de l'exécution de Louis XVI, la Terreur elle-même, qui leur semblent mettre en scène des épisodes illustres de l'histoire antique de la Grèce et de Rome -, que plutôt la longue crise directoriale, à partir de 1795, avec ses aspects internes (le double péril néo-jacobin et royaliste, les complots, la corruption) et externes (les guerres de conquête, l'exploitation des régions conquises, les débats sur la création de républiques-sœurs ou le rattachement pur et simple à la République, par exemple sur la rive gauche du Rhin). C'est cette longue crise, cette dégénérescence à l'intérieur même de la Révolution qui, d'un côté, alimente leur radicalisme, mais, d'un autre, le rend incertain et fragile ${ }^{40}$.

Un philosophe, Fichte, né en 1762 - chronologiquement, il tient le milieu entre la génération plus ancienne née autour de 1740-1750 et celle de 1770-, fournit à ces jeunes hommes, à Friedrich Schlegel surtout, mais aussi à Novalis, une part importante de leur arsenal intellectuel, essentiellement autour de ce que l'on peut appeler une théorisation de l'hypertrophie du Moi. Ses textes de 1793 sur la Révolution (Revendication de la Liberté de pensée et Contribution à la rectification des jugements du public) fondent sa réputation de «jacobin» que vient confirmer, en 1794, une Doctrine de la Science que Fichte lui-même appelle "le premier système de la liberté ", c'est-à-dire la théorie de la Révolution française au service de laquelle il souhaite se mettre personnellement. L'accusation d'athéisme portée contre lui un peu plus tard, en 1799, à l'université d'Iéna, est la conséquence de cette sulfureuse réputation de "jacobin ", mais aussi l'épilogue de cette fameuse "querelle du panthéisme ", et de l'athéisme, autour de l'interprétation du spinozisme et de la dernière pensée de Lessing, qui occupe bien des esprits allemands - Jacobi, Mendelssohn, Herder et Goethe, pour ne citer que les plus connus - depuis 1785 et pendant toute la dernière décennie du XVIII ${ }^{\mathrm{e}}$ siècle ${ }^{41}$. Par où l'on voit une fois encore comment des discussions en apparence très érudites et abstraites recoupent directement, comme aussi dans le cas des représentations de l'Antiquité, l'actualité politique la plus immédiate.

Friedrich Schlegel, Novalis et la référence germano-médiévale

37 Un phénomène étrange, inexplicable presque, se produit cependant au tournant du $\mathrm{XVIII}^{\mathrm{e}}$ au XIX ${ }^{\mathrm{e}}$ siècle. Malgré son "jacobinisme » intellectuel, malgré sa "grécomanie » qui n'est, selon Schiller, qu'une deuxième "gallomanie » révolutionnaire, Friedrich Schlegel, en l'espace de quelques années, passe de la gréco-gallomanie initiale à la germanomanie, qui est aussi la gallophobie des poèmes - par exemple sur la Wartburg ou sur le Rhin - et des textes en prose publiés à partir de 1803 dans la revue qu'il a fondée, Europa. C'est d'une véritable conversion au Moyen Âge germano-chrétien qu'il s'agit. Et pourtant, dans son livre sur la poésie grecque de 1795-1797, Schlegel n'avait que dédain pour cette période historique présentée comme un simple intervalle de barbarie entre l'Antiquité et le monde moderne «éclairé » - on pense au «monde vide 
depuis les Romains » de Saint-Just. Désormais, c'est l'image positive d'un Saint-Empire médiéval, hiérarchique, corporatif et chrétien qui est diffusée par Schlegel. Pour essayer de comprendre cet impressionnant tournant intellectuel et politique, peut-être faut-il revenir sur les textes antérieurs de Schlegel. Dès 1797, en effet, son éloge de Forster portait davantage sur les qualités humaines, scientifiques et littéraires du "jacobin" mayençais que sur ses idées et pratiques révolutionnaires. Quant aux Fragments des années 1798-1800, ils relativisaient fortement le républicanisme démocratique par l'adjonction d'éléments aristocratiques et monarchiques. Il est vrai que l'Essai sur le Concept de républicanisme, compte rendu du texte de Kant sur la paix de 1795 , proposait dès 1796 de décompter les voix, non d'après leur nombre mais d'après leur "poids». Quant aux Idées de 1800, elles affichaient la recherche d'un contrepoids spirituel à la Révolution française comme à l'Ancien Régime.

Dans les premières années du XIX ${ }^{e}$ siècle, la grandeur de l'Allemagne envisagée par Schlegel n'était plus celle esquissée par Schiller, en 1797, dans l'ébauche de poème intitulée, précisément, Grandeur allemande ${ }^{42}$ : à une grandeur schillérienne d'ordre simplement moral et intellectuel - le Saint-Empire étant considéré comme une structure irrémédiablement vermoulue - succédait, chez Schlegel, la vision d'une puissance bien plus pratique et politique dont le Saint-Empire médiéval fournissait le modèle idéal. Le Saint-Empire branlant et désuet de l'esquisse schillérienne prenait, régénéré par le jeune romantique - pourtant fort critique, quelques années plus tôt, à l'égard de la modération politique de son aîné et rival - les traits d'un certain avenir allemand.

39 Le tournant décisif du jeune Schlegel concerne deux éléments principaux : d'une part, l'orientation vers un modèle impérial, allemand et médiéval opposé au modèle précédent, révolutionnaire, français et antique d'un même mouvement ; d'autre part, une relativisation et une esthétisation du politique, particulièrement sensibles dans le célèbre aphorisme des Fragments, parus en 1798 dans la revue Athenäum, mettant en parallèle, comme grandes tendances représentatives de l'époque, la Révolution française, la Doctrine de la Science de Fichte et le roman Wilhelm Meister de Goethe, ainsi que dans l'étrange roman publié par Schlegel en 1799, Lucinde. Cette relativisation esthétisante du politique prend appui, surtout, sur une lecture de la pensée de Fichte et de son absolutisation du Moi dans le sens de l'arbitraire (Willkür) individuel et souverain, du jeu distant et ironique, destructeur en fin de compte, avec le réel, l'histoire, la société et la politique, ce jeu romantique que Hegel critiquera si souvent et si durement. Il est possible également que la présentation par Schiller, dans son texte sur l'éducation esthétique de 1795 , du libre jeu comme fondement de l'activité humaine, ait eu sa part dans ce processus intellectuel.

Quant au premier élément, plus immédiatement politique, de ce tournant décisif, celui du modèle impérial, médiéval et germano-chrétien, il a à l'évidence son origine principale, sinon unique, dans la lecture par les frères Schlegel de l'essai de Novalis $L a$ Chrétienté ou l'Europe, rédigé à l'automne 1799 et qui, sur le conseil de prudence de Goethe, qui le trouvait trop antifrançais et trop favorable au catholicisme, ne fut pas publié dans la revue Athenäum. Si la référence à la Grèce antique reste longtemps dominante chez Schlegel, comme elle l'était chez les intellectuels allemands de Winckelmann à Schiller, elle est faible et s'estompe rapidement chez son ami Novalis, lui aussi favorable, pourtant, en 1793-1794 - sa correspondance le montre -, à la phase 
la plus radicale de la Révolution française, et lui aussi marqué par Fichte et sa Doctrine de la Science, explicitement mentionnée vers la fin de La Chrétienté ou l'Europe.

Dans ce dernier texte, la référence dominante est constituée par la vision mythique d'un Moyen Âge chrétien harmonieux et beau - on pense irrésistiblement à l'image de la Grèce antique chez un Winckelmann, un Schiller ou un Forster - opposée à la malheureuse, triste et négative succession de la Réforme luthérienne, du rationalisme cartésien, des Lumières et de la Révolution française, la France apparaissant comme le lieu de concentration de toute cette négativité : tournant décisif, plus encore que celui de Schlegel, vers ce qui constitue une des bases majeures du romantisme politique allemand pendant tout le premier tiers du xixe siècle et même au-delà, jusqu'aux vues de Frédéric-Guillaume IV de Prusse et de son entourage, au début des années 1840, sur l'union des confessions chrétiennes séparées depuis la Réforme et sur un très désirable État germano-chrétien, rempart contre le libéralisme parlementaire et les revendications démocratiques. Et l'on sait que le conservatisme et la réaction allemands, jusqu'à nos jours, ont puissamment développé, y compris dans leur "modernité», cette référence germanique, chrétienne, médiévale et, au total, antifrançaise.

Ce tournant décisif de Schlegel, appuyé sur le texte à la fois fondateur et clandestin - sa première publication partielle n'aura lieu qu'en 1826 - de Novalis, est un tournant esthétique autant qu'un tournant politique. Ou, plus exactement, il s'agit d'un tournant esthétique au service d'une vision politique aussi subtilement que profondément réactionnaire, ce que confirment, au même moment ou à peu près, en 1797, les Épanchements d'un moine ami des arts de Wackenroder, lui aussi pourtant, en 1793, enthousiasmé par la Révolution française dans sa phase "terroriste", comme le montre sa correspondance avec son ami Ludwig Tieck.

Une autre génération de 1770 : Hegel et Hölderlin

43 Une question demeure, cependant, celle du lien exact - si lien il y a - entre le tournant du premier romantisme, au cours des années 1799-1803, vers la référence impériale, médiévale et germano-chrétienne opposée à la référence antérieure, antiquisante, révolutionnaire, ou "jacobine ", et païenne, ou antichrétienne, d'une part et, d'autre part, le tournant esthétisant, ironique et subjectiviste, celui du libre choix et du libre jeu de l'esprit avec le réel, amorcé dès 1796-1797. Cette question en inclut une autre, peut-être plus importante pour notre propos : comment la pensée de Fichte peut-elle être, à la fois, au fondement d'une approche de type révolutionnaire, « jacobin » si l'on préfère - et d'abord chez le philosophe lui-même - de la réalité politique, et au point de départ d'un tournant esthétique majeur, fondé sur l'absolutisation du Moi souverain, vers la relativisation et l'ironisation de la politique et de l'histoire, si caractéristiques du premier romantisme allemand autour de Friedrich Schlegel, ce tournant esthétique étant aussi, on l'a vu, un tournant politique?

Cette présence massive, complexe et assez énigmatique de la pensée fichtéenne, on la retrouve à l'origine, pour une part, de la carrière intellectuelle de deux représentants éminents de la très riche génération de 1770 , deux figures dont l'évolution contredit pourtant très largement celle du premier romantisme, deux amis de jeunesse nés l'un et l'autre en 1770 en Wurtemberg, condisciples d'un troisième ami de jeunesse, plus précoce et plus rapidement et efficacement organisé qu'eux, Schelling, au séminaire protestant (Stift) de Tubingue au début des années 1790 et plantant avec lui, à ce qu'on 
rapporte, un arbre de la liberté en l'honneur de la Révolution française : on aura bien sûr aisément reconnu Hegel et Hölderlin.

Hegel connaît certes, du préceptorat bernois à Francfort, entre 1793-1794 et 1800, une évolution importante qui, par étapes, le conduit de la critique rationaliste ou " éclairée ", antichrétienne et, conjointement, antidespotique de ses premiers écrits improprement appelés « théologiques » - autour de la notion significative, empruntée à la fois à la Grèce antique et à Rousseau, de " religion populaire » ("Volksreligion ») - aux textes des années 1798-1800, contemporains de La Chrétienté et l'Europe de Novalis et du tournant germano-chrétien de Schlegel et plus marqués par la représentation chrétienne de l'amour et par la recherche, dans le christianisme lui-même, du principe du mouvement historique ${ }^{43}$. Mais jamais ne seront vraiment reniées, chez Hegel, jusqu'aux années 1818-1831 du glorieux enseignement berlinois, en particulier sur la philosophie de l'histoire et l'esthétique, d'une part, la dure critique du subjectivisme et de l'ironie romantiques à la Schlegel, débouchant sur le vide moral et la destruction du sérieux du monde réel et historique, d'autre part, la double et émouvante fidélité, pardelà les tristes et grises réalités de la Restauration allemande et européenne, à l'image idéale, schillérienne et même forstérienne, de la Grèce classique, belle, heureuse et libre, et à l'enthousiasme du jeune homme pour une Révolution française - le «splendide lever de soleil »- qui, un temps, a pu paraitre capable de faire revivre, ou vivre cette Grèce idéale dans le monde moderne, même si Hegel sait et répète sur différents tons que l'art grec antique, comme la cité athénienne démocratique, constituent, à la fois, un indépassable sommet dans l'évolution de l'humanité et un idéal d'harmonie auquel il est vain de penser pouvoir revenir ${ }^{44}$. En disant et en écrivant cela, Hegel reste proche des conceptions d'un Schiller ou d'un Herder, même s'il s'éloigne quelque peu du radicalisme «jacobin » de Forster ou, de façon - on l'a vu infiniment plus problématique et fragile, de Friedrich Schlegel.

Quant à Hölderlin, qui partage ces idéaux, ces nostalgies et ces fidélités avec Hegel, il n'est certes pas, à la différence de son ami, l'homme des résignations lucides et des accommodements progressifs, le théoricien de ce qui est, effectivement, et qui n'est pas forcément ce que l'on a souhaité voir être. Mais il n'est pas davantage, à la différence de ses très exacts contemporains de la jeune génération romantique - Friedrich Schlegel particulièrement -, l'homme des tournants intellectuels brusques, des virages politiques pris par opportunisme - que l'on pense aux soucis d'argent des frères Schlegel ! - ou par conviction, ou pour les deux raisons à la fois. D'où sans doute, à la fin des années 1790 , l'échec de sa tentative de rapprochement avec Schiller ${ }^{45}$, d'où aussi l'échec de sa tentative pour fonder une revue, Iduna, en 1798-1799, au moment même où les frères Schlegel publient leur Athenäum ${ }^{46}$, d'où enfin l'absence d'écho critique favorable à son roman Hypérion en 1797-1799 auprès des premiers romantiques, à l'exception notable d'une élogieuse recension de Joseph Görres, en 1804. Mais Görres, l'ancien «jacobin» cisrhénan, même converti au romantisme national et au conservatisme catholique, en apparence au moins, a-t-il jamais été un romantique de stricte obédience ${ }^{47}$ ? L'opposition entre Hölderlin et la première génération du romantisme allemand n'est nulle part plus sensible, nous semble-t-il, que dans l'opposition entre le cinquième des Hymnes à la Nuit de Novalis, en 1800, dans lequel le Christ signifie et manifeste une rupture absolue avec la lignée des dieux de la Grèce antique, leur mort, et l'élégie Le Pain et le Vin de Hölderlin, en 1800-1801, dans laquelle 
le même Christ apparaît comme le dernier représentant de cette même lignée, son achèvement ou son accomplissement.

Conclusion

47 L'étude dont quelques jalons ont été posés ci-dessus fait apparaître, dans les lettres et la pensée allemandes de la deuxième moitié $\mathrm{du} \mathrm{xVIII}^{\mathrm{e}}$ siècle, l'existence d'une ligne intellectuelle continue et puissante, de Winckelmann au jeune Friedrich Schlegel, à Hölderlin et à Hegel, en passant par Heinse, Herder et Forster, marquée par l'intérêt à la fois artistique et érudit pour l'Antiquité, grecque surtout, et par l'affirmation d'un lien de consubstantialité entre la beauté (naturelle et artistique), le bonheur (individuel et collectif) et la liberté (du citoyen et de la cité), les uns et les autres désignés comme les héritages revivifiés, ou à faire revivre dans le monde moderne, des formes, des pensées et des pratiques antiques.

Cette ligne intellectuelle se trouve réactivée, à la fin du siècle, par une Révolution française qui semble en mesure - c'est là l'» illusion héroïque » diagnostiquée par le jeune Marx et par bien des marxistes après lui- de faire renaître dans le monde moderne, celui de la triste " prose bourgeoise » dont parlera plus tard Hegel, la belle liberté et le poétique bonheur des Grecs anciens, malgré les réserves formulées, surtout à partir de 1792-1793, en particulier par Schiller et, plus généralement, par ce qu'on appelle le "classicisme allemand", à l'époque de la collaboration amicale et active entre Goethe et Schiller, de 1794 à 1805, année de la mort du second. Ce classicisme-là, adoptant une position en surplomb sur les débats politiques et intellectuels du moment, marque un mouvement d'hésitation, ou de relatif retrait de la pensée face au réel historique, la recherche d'un "point d'indifférence " ("Indifferenzpunkt »)l'expression sera utilisée par Heine, à propos de Goethe, dans ses écrits sur l'Allemagne du début des années 1830 - supérieur d'où décrire et éventuellement juger l'époque, ses acteurs et ses commentateurs littéraires et philosophiques.

Face à cette ligne intellectuelle longtemps largement dominante, avec ses variantes modérées ou radicales, se fait jour, au cours des dernières années du xviIIe siècle et au tout début $\mathrm{du} x \mathrm{xIX}^{\mathrm{e}}$, autour du premier romantisme allemand en voie de constitution (Novalis, les frères Schlegel - en particulier le jeune Friedrich Schlegel deuxième manière -, Wackenroder et Tieck), la réactivation d'une référence médiévale et germanique - ou supposée telle, par exemple à propos de l'art "gothique » - déjà présente au début des années 1770 (le premier Herder, le jeune Goethe à Strasbourg) dans le mouvement littéraire du Sturm und Drang. Mais, alors que celui-ci avait surtout le sens d'une radicalisation, y compris politique, de l'Aufklärung, les Lumières allemandes, une signification prérévolutionnaire, d'un point de vue téléologique, le tournant décisif du premier romantisme a, d'emblée, et plus encore dans ses suites - la carrière personnelle et intellectuelle de Friedrich Schlegel le montre ${ }^{48}-$, jusqu'aux années 1830 et 1840 au moins, le sens d'une réaction germano-chrétienne à la fois intellectuelle et politique contre la Révolution française, ses idéaux, ses acquis pratiques et, plus généralement, un libéralisme et un radicalisme démocratique souvent identifiés au voisin occidental, la France.

Deux aspects de ce vaste débat récurrent nous semblent particulièrement complexes, voire énigmatiques. Le premier est celui de la fonction précise de la pensée de Fichte dans la genèse du premier romantisme. Si son influence est incontestable sur Friedrich Schlegel, et même sur Novalis, comment expliquer qu'une pensée qui se donne ellemême, explicitement, pour révolutionnaire, et même, à proprement parler, pour une 
pensée de la Révolution, au service de celle-ci, puisse aboutir, dans certains de ses effets, à un tournant visiblement réactionnaire, au plein sens du mot, dans la pensée et les lettres allemandes ? La réponse à cette question doit, à notre avis, être recherchée du côté de cette hypertrophie théorique du Moi qui caractérise la pensée de Fichte, avec comme conséquences le libre jeu de l'intellect avec le réel et, finalement, l'idéalisme et l'esthétisme subjectifs les plus radicaux, si vigoureusement critiqués par Hegel, tenant de l'idéalisme objectif. Le premier romantisme allemand et son tournant germano-chrétien ne sont certes pas le seul exemple d'une pensée - en l'occurrence celle de Fichte - révolutionnaire, ou se donnant pour telle, retournée en son contraire à la fois par le jeu de l'esprit et les contraintes de l'époque : la réaction intellectuelle et politique.

51 Le deuxième aspect susceptible d'appeler la discussion est celui de la genèse, au fil de ce long débat sur l'Antiquité, la Révolution française et leurs contraires - le Moyen Âge chrétien supposé germanique et la réaction politique, ou la contre-révolution-, du patriotisme ou du nationalisme allemands. Il y a bien, de Winckelmann à Hölderlin et au jeune Hegel, mais aussi chez le jeune Friedrich Schlegel dans sa première manière, "gallo-grécomane ", l'affirmation - qui n'est pas exclusivement culturelle, même si elle l'est d'abord et principalement - d'un sentiment patriotique allemand. Mais cette affirmation-là, variable dans son intensité et ses modalités, conserve toujours, suivant l'exemple donné par Herder dans ses écrits sur la philosophie de l'histoire, une orientation et une valeur universalistes. Elle reste explicitement compatible, en règle générale, avec l'affirmation, au même moment, du patriotisme chez les nations voisines : le patriotisme, l'amour actif du citoyen libre pour sa cité libre, n'est-il pas, lui aussi, un héritage de l'Antiquité grecque et romaine, lui aussi puissamment réactivé et de quelle manière ! - par la Révolution française ? Le tournant décisif du romantisme allemand au tout début du XIX ${ }^{e}$ siècle, puis à partir de 1806, avec la fin du Saint-Empire, et plus encore au moment des guerres antinapoléoniennes dites "de libération" (" Befreiungskriege ") de 1813-1815, confère - relayé et vulgarisé par les idéologues de la teutomanie la plus arrogante comme Arndt ou Jahn - au patriotisme allemand une orientation nouvelle, plus exclusive, parfois totalitaire dans ses expressions les plus radicales, l'antisémitisme de certains romantiques (Achim von Arnim par exemple) et des idéologues $\mathrm{du}$ mouvement national rejoignant indissolublement, consubstantiellement, leur francophobie, voire leur francophagie. Mais c'est là déjà une autre histoire, et qui va loin.

\section{NOTES}

1.Georg LUKACs, Ausgewählte Schriften II, Reinbek bei Hambourg, 1967, pp. 88 sqq.

2.Cité ibid. p. 78. Trad. française in : Karl MARX, Euvres, III sous la resp. de Maximilien RUBEL, Paris, 1982, p. 561.

3.Voir l'introduction de Claude Prévost aux Écrits de Moscou, Paris, 1974, pp. 7-59.

4.Lucien GoldmanN, Sciences Humaines et Philosophie, Paris, 1966, p. 100. 
5.Pierre BERTAUX, Hölderlin und die Französische Revolution, Frankfurt-am-Main, 1969, p. 141.

6.Ibid., p. 49.

7.Voir en particulier Claude MossÉ, L'Antiquité dans la Révolution française, Paris, 1989 ; Pierre VIDAL-NAQUET, : “Tradition de la démocratie grecque”, dans Moses I.Finley, Démocratie antique et démocratie moderne, Paris, 1990, pp. 7-44 ; La Grecia antica. Mito e simbolo per l'età della Grande Rivoluzione, a cura di P. Boutry, P. Chiarini, etc., Milan, 1991 et Philippe BORDES, La Mort de Brutus de Pierre-Narcisse Guérin, publié à l'occasion de l'exposition “Lucius Junius Brutus. L’Antiquité et la Révolution française”, Musée de la Révolution française, Vizille, 1996.

8.Michael hofmAnN, : "Conscience de crise historique et poétique de la modernité : Wallenstein de F. Schiller", dans Crise et Conscience du temps, dir. J.-M. Paul, Nancy, 1998, p. 132. Sur le rapport du classicisme allemand à l'Antiquité et à la Révolution, la littérature est abondante. Voir en particulier BAIONI, Giuliano, Goethe. Classicismo e Rivoluzione, Turin, 1998 (première éd. en 1969).

9.La littérature sur les réactions à la Révolution française en pays allemand est très riche. Signalons seulement, en français, Marita GILLI, Pensée et Pratique révolutionnaires à la fin du XVIIIe siècle en Allemagne, Besançon et Paris, 1983 et La Révolution française vue par les Allemands, textes trad. et prés. par Joël Lefebvre, Lyon, 1987.

10.Jean STAROBINSKY, 1789, les Emblèmes de la raison, Paris, 1979, p. 5.

11.Hugh hONOUR, Le Néo-classicisme, trad. de l'anglais par P.-E. Dauzat, Paris, 1998, pp. 88-89.

12.Ibid., p. 199.

13.Georg LUKACS, Ausgewählte Schriften II, op. cit. p. 78. En l'absence d'indication du traducteur ou du responsable de la trad., les traductions de l'allemand sont de notre fait.

14.WINCKELMANN, Werke, Berlin et Weimar, 1982, p. 7.

15.Ibid., pp. 180 et 182 .

16. HEINSE, Ardinghello, trad. de l'allemand par A. Jolivet, Paris, 1944, p. 178.

17.Ibid., p. 173.

18.Ibid., p. 174.

19.Ibid.

20.Ibid., p. 175.

21.Rapport à la Convention du 31 mars 1794 (Saint-Just, Euvres choisies, Paris, 1968, p. 249).

22.Schiller und sein Kreis, hrsg. von O. Fambach, Berlin, 1957, p. 48.

23.Ibid., pp. 60-68.

24.Griechenland als Ideal, hrsg. von L. Uhlig, Tübingen, 1988, p. 72.

25.SCHILLE, Lettres sur l'Éducation esthétique, trad. de l'allemand par R. Leroux, Paris, 1943, p. 119.

26.Georg LUKACS, Ausgewählte Schriften II, op.cit., pp. 88-89.

27.HERDER, Werke, Berlin et Weimar, 1978, II, pp. 336-346.

28.Ibid., III, p. 57.

29.Ibid.

30.Ibid., IV, p. 271.

31.Ibid., pp. 278-279.

32.Ibid., p. 298. 
33.SCHLEGEL, Kritische Schriften, Munich, 1964, p. 323.

34.Voir Inge STEPHAN, Literarischer Jakobinismus in Deutschland, Stuttgart, 1976, p. 122.

35. Cité ibid.

36. Cité par Georg LUKACS, Ausgewählte Schriften II, op. cit., p. 89.

37.Voir Gerda HEINRICH, Geschichtsphilosophische Positionen der deutschen Frühromantik, Berlin, 1976, en part. pp. 81-100.

38.SCHLEGEL, Kritische Schriften, op. cit., pp. 113-230.

39.Voir Lucien CALVIÉ, “Jacobinisme et idée nationale en Allemagne à l'époque révolutionnaire : le cas de Görres", Annales Historiques de la Révolution française, octobredécembre 1990, pp. 404-421.

40.Voir Lucien CALVIÉ, "La génération de 1770 et le premier romantisme allemand : crise et conscience de crise", dans Crise et Conscience du temps, dir. J.-M. Paul, Nancy, 1998, pp. 97-110.

41.Sur l'accusation d'athéisme, voir Xavier LÉon, Fichte et son temps, Paris, 1922-1924, I, pp. 518-630.

42.SCHILLER, Sämtliche Werke, Munich, 1965-1966, II, pp. 226-229.

43.Voir André KAAN, “Introduction”, dans HEGEL, Le Droit naturel, trad. de l'allemand par A. Kaan, Paris, 1972, pp. 6-14.

44.Voir Kostas PAPAIOANnOU, Hegel, Paris, 1987 (première édition, Paris, 1962), en particulier pp.30-38.

45.Voir Günter MIETH : "F. Hölderlin und F. Schiller - Die Tragik einer literaturgeschichtlichen Konstellation”, Hölderlin-Jahrbuch, 1992-1993, pp. 68-79.

46.Voir Roger AYRAULT : "Hölderlin et les premiers romantiques. Sur le projet de revue Iduna en 1799", Revue d'Allemagne, octobre-décembre 1977 (Hommage à Pierre Bertaux), pp. 571-588.

47.Voir Lucien CALVIÉ : “Le roman Hypérion de Hölderlin et l'Histoire”, Chroniques allemandes, 1, 1992, pp. 89-106.

48. Voir Joël LEFEBVRE : “Du républicanisme à la Restauration. L'évolution de la pensée politique de F. Schlegel”, dans La Révolution française dans la pensée européenne, dir. D. Schulthess et P. Muller, Neuchâtel et Lausanne, 1989, pp. 101-125.

\section{RÉSUMÉS}

À la fin du XVIII ${ }^{\mathrm{e}}$ siècle, le discours allemand sur l'Antiquité est, en deuxième instance, un discours sur la Révolution : la bourgeoisie allemande, par la voix d'écrivains comme Schiller ou Hölderlin, «parle antique» parce qu'elle est trop faible pour "parler allemand», c'est-à-dire pour constituer son État national. À l'affirmation à la fois révolutionnaire et antiquisante d'un lien de consubstantialité entre la beauté, le bonheur et la liberté, le premier romantisme (Novalis) oppose la réactivation, à proprement parler réactionnaire, d'une référence germanique et médiévale.

Antiquity and the French Revolution in German Thought and Letters at the Close of the Eighteenth Century. At the close of the eighteenth century, German discourse on antiquity was 
at one remove a discourse on the Revolution: the German bourgeoisie, through the agency of writers like Schiller and Hölderlin, resorted to classical terms because they were too weak to use the German idiom, i. e. to build their own nation-state. In opposition to revolutionary and antiquity-orientated claims of a consubstantial link between beauty, happiness and liberty, the early Romantics (Novalis) called forth the somewhat reactionary reference model of a Germanic and medieval past.

Alla fine de Settecento, il discorso tedesco sull'Antichità è anche discorso sulla Rivoluzione : la borghesia tedesca, usando la voce di scrittori come Schiller o Hölderlin, «parla antico " perché è troppo debole per " parlare tedesco », cioè par costituire il suo Stato nazionale. All'affermazione, allo stesso tempo rivouzionario ed antichizzante d'un legame fondamentale tra bellezza, felicità et libertà, il primo romanticismo (Novalis).

Al final del siglo XVIII, el discurso germano sobre la Antigüedad es también un discurso sobre la Revolución: demasiado debil para "hablar germano", es decir para constituir un Estado nacional, la burguesía germana «habla antiguo» por medio de escritores como schiller o Hölderlin. A la afirmación, a la vez revolucionaria y de raiz antigua, de un lazo de consubstancialidad entre la belleza, la felicidad y la libertad, el primer romanticismo (Novalis) opone la reactivación, hablando con propiedad reaccionaria, de una referencia germánica y medieval.

Am Ende des 18. Jahrhunderts ist der deutsche Diskurs über die Antike in zweiter Ebene ein Diskurs über die französische Revolution: Durch das Sprachrohr von Schriftstellern wie Schiller und Hölderlin spricht das deutsche Bürgertum von der Antike, weil es zu schwach ist, um von Deutschland zu sprechen, d. h. zu schwach, um seinen Nationalstaat zu bilden. Dieser zugleich revolutionären und antikisierenden Behauptung einer gleichen Wesensart zwischen der Schönheit, der Glückseligkeit und der Freiheit setzt die erste romantische Schule (Novalis) die eigentlich reaktionäre Reaktivierung eines Hinweises auf ein deutsches Mittelalter entgegen.

\section{AUTEUR}

\section{LUCIEN CALVIÉ}

Université Toulouse II - Le Mirail. 